ARTÍCULOS ORIGINALES Rev Chil Salud Pública 2014; Vol 18 (1): $72-80$

\section{INCORPORACIÓN DE LOS ODONTÓLOGOS EN LOS PROCESOS DE TRANSFORMACIÓN AL MODELO DE SALUD FAMILIAR DE LA ATENCION PRIMARIA EN CHILE}

\author{
Inclusion of Dentists in the transition to the Family \\ Health Care Model of Primary Care in Chile
}

\title{
RESUMEN
}

Objetivo: Describir la incorporación de los odontólogos en la implementación del modelo de salud familiar de la atención primaria.

Metodología: Se realizó estudio descriptivo de corte transversal, mediante encuesta enviada vía e-mail a 183 jefes de programas odontológicos de CESFAM acreditados del país.

Resultados: El 94\% de los equipos tiene a lo menos un odontólogo capacitado en relación al modelo; de ellos solo un $37 \%$ lo ha hecho mediante un diplomado de Salud Familiar, el resto ha realizado cursos o jornadas de capacitación en su establecimiento. Con respecto a la participación en actividades que ofrece el modelo, se observa una autopercepción positiva, la que disminuye con respecto a la incorporación de otros profesionales de la salud en los programas odontológicos o en la realización de nuevas prestaciones (14\% realiza consejerías familiares, $33 \%$ no realiza atención sectorizada). El $83 \%$ de los equipos realiza actividades de promoción y prevención en establecimientos educacionales; sin embargo se desaprovechan instancias que permitirian ejecutar el programa en forma integral (46\% participa del pro-

JAIME FARFÁN

Odontólogo, Magíster Salud Pública jaime.farfan@gmail.com

María Teresa VALENZUELA Magíster Salud Pública, Escuela Salud Pública Universidad de Chile grama "Chile Crece Contigo" y solo $15 \%$ en comités "Vida Sana").

Discusión: Se observa un alto porcentaje de capacitación de los equipos encuestados, que difieren en el tipo de formación y carga académica. Además, la alta participación en las actividades que ofrece el modelo no se ve reflejada en la integración de temas de salud bucal en ellas. Por último, se genera la necesidad de que a nivel central se formulen protocolos que permitan a los equipos odontológicos programar y ejecutar nuevas estrategias cuantificando su rendimiento.

Palabras clave: salud familiar, atención primaria, odontólogos. 


\section{ABSTRACT}

Objetive: To describe the inclusion of dentists in the implementation of the family bealth model in primary bealth care in Chile.

Methodology: We performed a cross-sectional descriptive study, using a survey sent via e-mail to 183 heads of accredited CESFAM (Centro de Salud Familiar) dental programs in the country.

Results: $94 \%$ of the teams have at least one dentist trained in the model, but only $37 \%$ bave obtained the Family Health Diploma certification; the rest have been trained through courses or training days at their workplaces. With regards to participation in activities related to the family bealth model, their perceptions are positive, and decrease with respect to the inclusion of other bealth professionals in the dental programs or including new features (14\% do family counseling, $33 \%$ do not practice sectorized care). $83 \%$ of the health teams do advocacy and prevention in educational institutions, but they do not take advantage of opportunities for comprehensive care (46\% of the program participates in "Chile Crece Contigo" and only $15 \%$ in "Vida Sana" community councils).

Discussion: There is a high proportion of respondents, which differ according to type and academic load. The high participation in activities offered by the model is not the integration of oral health issues in bealth teams. Finally, it shows the need for centralized protocols that allow dental teams to schedule and execute new strategies to improve their performance.

Keywords: Family Health, Primary Care, Dentists.

\section{INTRODUCCIÓN}

En los últimos diez años los centros de atención primaria de salud (APS) en Chile han aplicado un nuevo modelo de atención que pone a la familia como centro de las acciones para mejorar la salud de las personas (1). Su implementación obliga a iniciar una transformación en diferentes ámbitos, reflejada en la incorporación de nuevas prestaciones a las carteras de servicios de los centros asistenciales, como consejería familiar y visitas domiciliarias integrales, e iniciar procesos de cambios y fortalecimientos de los equipos de salud primaria, que incluyen actividades como diagnóstico de salud, implementación de normas, guías clínicas y objetivos comunes, con el fin de dar solución a los distintos problemas de salud de la población $(2,3)$. Entre los problemas que intenta resolver la APS se encuentran las patologías bucales, focalizando los recursos humanos y físicos a determinados grupos estratégicos; que incluyen a niños de 2, 4, 6 y 12 años, embarazadas y adultos de 60 años (4-6). Sin embargo, la baja cobertura de los programas de la primera infancia, entre otras razones, hace que muchas veces el primer contacto que tiene el odontólogo con el niño y su madre, sea generalmente a los 6 años, edad en que las patologías bucales, principalmente caries, ya están instauradas y avanzadas.

Frente a esta situación, se han hecho esfuerzos para actualizar las orientaciones programáticas, como en el caso del Control Odontológico del Niño Sano y la creación del Programa de Promoción y Prevención para niños y niñas preescolares, orientando las acciones principalmente a lo promocional y preventivo e incorporando una serie de intervenciones que obedecen a la necesidad de actuar en todas las etapas del ciclo vital, con el fin de lograr hábitos adecuados, utilizando a la familia como agente promotor junto con la participación de otros sectores en el cumplimiento de los objetivos finales de mejorar la salud bucal $(7,8)$.

Sin embargo, a pesar de las restructuraciones para abordar el problema desde una perspectiva integral con enfoque familiar, no 
existe suficiente evidencia que permita establecer, por ejemplo, el grado de capacitación en salud familiar o la cantidad de centros con atención odontológica sectorizada.

En este contexto, el objetivo del estudio fue describir el grado de incorporación de los odontólogos al modelo con enfoque familiar y comunitario que se está adoptando en la APS, estableciendo la situación actual y la real integración de las nuevas herramientas y conceptos, así como también su participación en los distintos equipos y programas multidisciplinarios que genera la implementación del nuevo modelo. Partimos de la base que un enfoque familiar en las prestaciones odontológicas permitiría abordar y solucionar de mejor forma los problemas de salud bucal, tal como lo demuestran diversas estrategias exitosas.

\section{MATERIAL Y MÉTODO}

Se realizó un estudio descriptivo transversal, considerando como marco muestral a los odontólogos jefes de programa que se desempeñan en los Centros de Salud Familiar (CESFAM) acreditados, quienes fueron los encargados de describir las actividades que desarrollan en conjunto. Se utilizó un listado con la información actualizada de 299 jefes de programas de 24 servicios de salud (correspondientes al $72 \%$ de establecimientos acreditados del país), obtenida mediante una solicitud de participación enviada por el Departamento de Salud Bucal del Ministerio de Salud, a los distintos asesores odontológicos. La recolección de los datos se realizó mediante la aplicación de una encuesta, la que fue sometida a una validez de contenido por un panel de expertos utilizando una evaluación cuantitativa (9). Finalmente se obtuvo una encuesta con un total de 19 preguntas cerradas y dos abiertas, la que evaluó cuatro dimensiones:

1. Grado de capacitación en relación al modelo de salud familiar.

2. Incorporación a las actividades en equipo del nuevo modelo.
3. Participación en actividades intersectoriales e interdisciplinarias que incluye el modelo.

4. Fortalezas y debilidades percibidas en la implementación del modelo.

Además se registró: Servicio de Salud, financiamiento per cápita comunal, número de profesionales por equipo y tiempo de acreditación del establecimiento.

La encuesta fue probada en 25 odontólogos que se desempeñaban en otros centros de atención primaria. Con el instrumento terminado y con el apoyo del Departamento de Salud Bucal del Ministerio, se envió vía e-mail a través del programa Google Docs a los jefes de programas, durante los meses de febrero y marzo de 2012. En el caso de no obtener respuesta, se contactó en forma telefónica con el fin de incentivarlos a responder.

Las respuestas obtenidas fueron operacionalizadas en distintos tipos de variables y resumidas, utilizando planillas Excel, para luego realizar el análisis estadístico con el programa Stata 8 y SPSS 18.0.

\section{RESULTADOS}

Se obtuvo respuesta de 183 jefes de programas, provenientes de 24 servicios de salud, una tasa de respuesta del $61 \%$. Al no existir investigaciones que permitan establecer el grado de participación de los odontólogos en el nuevo modelo, y considerando que la participación en programas de capacitación en salud familiar alcanza un $30 \%$ (10), los resultados obtenidos permiten tener una precisión del $5 \%$, considerando un intervalo de confianza del $95 \%$.

Los jefes de programas encuestados provenían en su mayoría de CESFAM pertenecientes a comunas con financiamiento per cápita urbanos; $66 \%(\mathrm{n}=121)$, seguidos por comunas rurales $31 \%(n=56)$ y un $3 \%(n=6)$ por aquellas que poseen mayor grado de ruralidad y pobreza (costo fijo). Esta distribución es similar a la observada en el total de CESFAM del país. Además la mayoría de los establecimientos encuestados estaba acreditado 
como CESFAM entre 2 a 5 años $51 \%$ ( $\mathrm{n}=93$ ), seguido por aquellos que lo estaban por más de 5 años $33 \%(n=61)$ y el $16 \%(n=29)$ con menos de 2 años. Con respecto a los 116 centros que no participaron de la encuesta, hubo una distribución similar en relación al per cápita comunal, tipo de servicio y tiempo de acreditación observado en la muestra.

En relación al porcentaje de capacitación en salud familiar, y considerando a todos los integrantes de los equipos encuestados, en la Tabla 1 se observa que los conformados con un nivel de capacitación mayor al $75 \%$ representan el $48 \%(n=87)$, mientras que solo el $6 \%(\mathrm{n}=11)$ no tiene odontólogos capacitados, tendencia que se repite en relación a las asistentes dentales. Sin embargo, al considerar el total de profesionales que forman parte de los equipos odontológicos, el 37\% ( $\mathrm{n}=337)$ de los odontólogos no ha recibido capacitación.

Con respecto al tipo de capacitación y considerando al profesional con el más alto nivel de capacitación por equipo encuestado, el 34\% ( $n=63$ ) de los odontólogos la realizó mediante un diplomado de Salud Familiar; el resto a través de cursos dictados por su establecimiento o por alguna universidad. Por otra parte, un $49 \%(n=90)$ de asistentes dentales recibió una capacitación en su establecimiento y solo un $1 \%$ realizó algún diplomado (Tabla 1).

En relación a la incorporación en actividades que ofrece el nuevo modelo, se observa que el $66 \%(n=120)$ realiza una atención odontológica sectorizada, mientras que en la ejecución de nuevas prestaciones, el $81 \%$ $(\mathrm{n}=148)$ realiza consejería individual; $44 \%$ $(\mathrm{n}=81)$, trabajo comunitario; $32 \%(\mathrm{n}=58)$, visita domiciliaria y solo un $14 \%(n=25)$, consejería familiar. En este contexto se observa que los equipos formados por odontólogos con un mayor grado de capacitación realizan un mayor porcentaje de estas prestaciones (Gráfico 2).

Con respecto a la incorporación en actividades interdisciplinarias e intersectoriales, se observa una buena autopercepción de los jefes de programas, donde el $91 \%(n=167)$ considera que siempre o casi siempre participan de las reuniones de sector y $94 \%(n=173)$ considera

Tabla 1: Grado de capacitación en salud familiar de los equipos odontológicos encuestados.

\begin{tabular}{|c|c|c|}
\hline Cantidad de profesionales por equipo & Odontólogos & Asistentes \\
\hline Capacitados & $567(63 \%)$ & $539(89 \%)$ \\
\hline No capacitados & $337(37 \%)$ & $245(31 \%)$ \\
\hline Total & $904(100 \%)$ & $784(100 \%)$ \\
\hline Porcentaje de capacitación por equipo & Odontólogos & Asistentes \\
\hline Ninguno & $11(6 \%)$ & $18(10 \%)$ \\
\hline Menor al 25\% & $9(5 \%)$ & $6(4 \%)$ \\
\hline Entre $25 \%$ y $50 \%$ & $30(16 \%)$ & $15(8 \%)$ \\
\hline Entre $50 \%$ y $75 \%$ & $46(25 \%)$ & $44(25 \%)$ \\
\hline Mayor al $75 \%$ & $87(48 \%)$ & $100(55 \%)$ \\
\hline Total & $183(100 \%)$ & $183(100 \%)$ \\
\hline \multicolumn{3}{|l|}{$\begin{array}{l}\text { Tipo de capacitación (Considerando al profesional } \\
\text { con el más alto nivel de formación) }\end{array}$} \\
\hline Ninguna capacitación & $11(6 \%)$ & $18(10 \%)$ \\
\hline Diplomado salud familiar & $63(34 \%)$ & $2(1 \%)$ \\
\hline Pasantías nacionales o internacionales & $11(6 \%)$ & $17(9 \%)$ \\
\hline Curso dictado por universidades & $31(17 \%)$ & $19(11 \%)$ \\
\hline Curso dictado en el servicio de salud & $22(12 \%)$ & $37(20 \%)$ \\
\hline Jornada de capacitación en su establecimiento & $45(25 \%)$ & $90(49 \%)$ \\
\hline Total & $183(100 \%)$ & $183(100 \%)$ \\
\hline
\end{tabular}


adecuada la derivación y coordinación de pacientes. Sin embargo, esta participación disminuye en la incorporación y programación de temas de salud bucal por parte de los equipos odontológicos (Gráfico 3). Se observa una alta participación en actividades comunitarias, destacando que un $83 \%$ lo hace en colegios y jardines infantiles comunales, seguido por actividades desarrolladas por otros medios o programas (ver Gráfico 3).

Frente a los beneficios que puede otorgar la incorporación del programa odontológico al nuevo modelo, la mayoría coincide en que se traduciría en un enfoque preventivo y promocional, integral y de trabajo en equipo ya que entre el $84 \%(n=154)$ y el $79 \%(n=145)$ de los equipos encuestados se inclinaron por esas respuestas. En relación a las barreras observadas por los equipos encuestados para incorporar este nuevo modelo, la mayoría coincide en las horas de odontólogos insuficientes $(74 \%)$, seguido por la falta de recursos físicos (49\%), y que el odontólogo no es invitado a participar de las actividades (32\%).

Por último, en relación a los cambios que se pueden realizar para incorporar el enfoque familiar al programa odontológico, la mayoría coincide en que se debe disponer de protocolos estandarizados para compartir con el equipo $(70 \%)$, seguido por una mejor coordinación con los establecimientos (48\%).

\section{DISCUSIÓN}

Se obtuvo un total de 183 centros encuestados, cuya distribución según servicio de salud, tipo de financiamiento per cápita y tiempo de acreditación genera que los resultados obtenidos tengan una representatividad adecuada y reflejen la situación de establecimientos que llevan años adoptando los lineamientos de este nuevo modelo.

Con respecto a la capacitación en salud familiar de los equipos encuestados, se genera una desventaja en la implementación de estrategias o prestaciones odontológicas con enfoque familiar, debido al desconocimiento de estas o no poseer los fundamentos teóricos y prácticos que pueden ser adquiridos mediante una capacitación de mayor significancia como un diplomado, versus un curso dictado por un establecimiento. Además, una mayor capacitación en salud familiar genera mejores resultados sanitarios, llegando a provocar una disminución de la mortalidad en todas sus causas y un bajo número de hospitalizaciones y consultas de urgencias (11). Situación

Gráfico 1: Porcentaje de prestaciones con enfoque familiar y comunitario que desarrollan los equipos odontológicos encuestados, según tipo de capacitación del odontólogo.

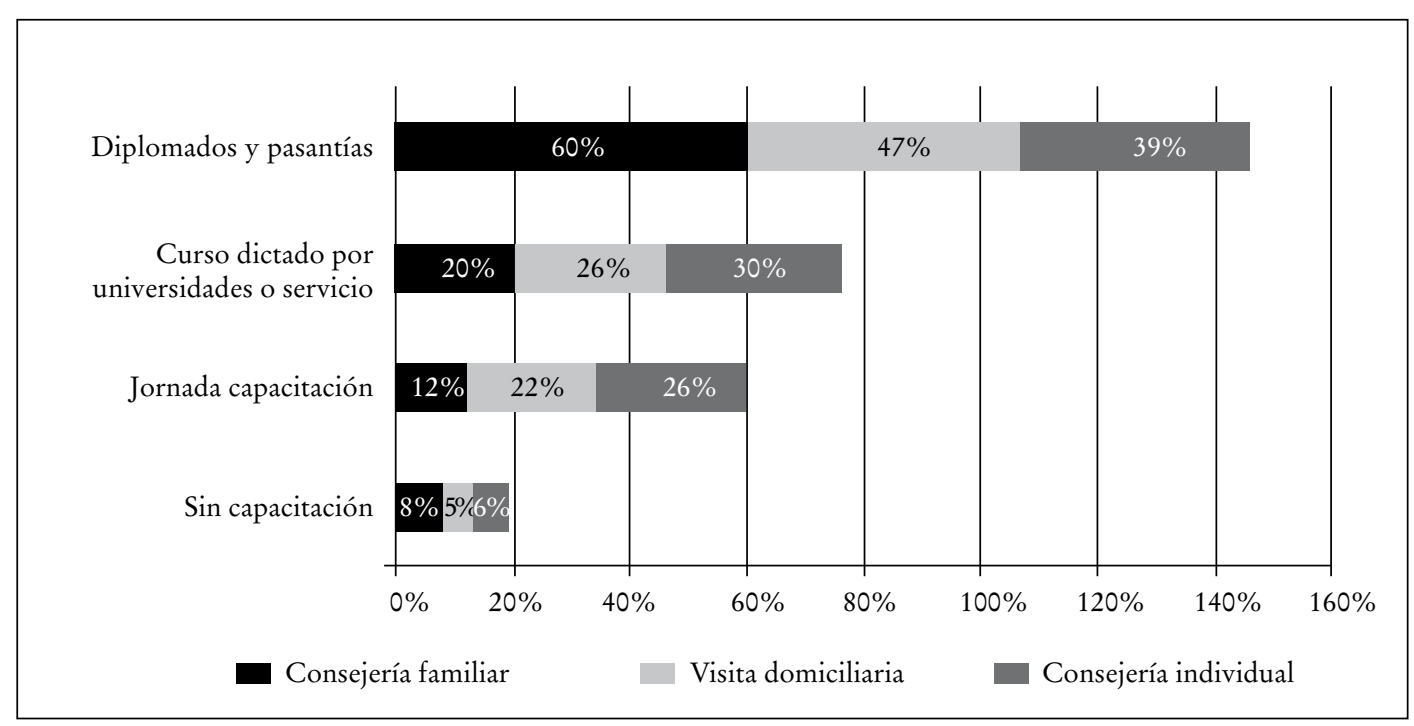


compartida al comparar los centros de salud Ancora de la Universidad Católica de Chile (establecimientos dotados con un $30 \%$ a un $60 \%$ de médicos especialistas en salud familiar y con un per cápita especial) con otros centros municipalizados, observándose una mayor eficacia de los centros universitarios y una menor tasa de derivación a niveles de mayor complejidad. Sin embargo, estas interven- ciones significaron una inversión mayor para capacitar a sus profesionales, pero que genera un costo-beneficio adecuado de 0,6 dólares por cada punto de efectividad ganado $(12,13)$.

En relación a la incorporación en actividades que ofrece el modelo, se observa una gran participación en las reuniones de sector o del equipo gestor en sus respectivos CESFAM. Sin embargo, esta alta participación no se materia-

Gráfico 2: Autopercepción en la incorporación de actividades interdisciplinarias de los equipos odontológicos encuestados.

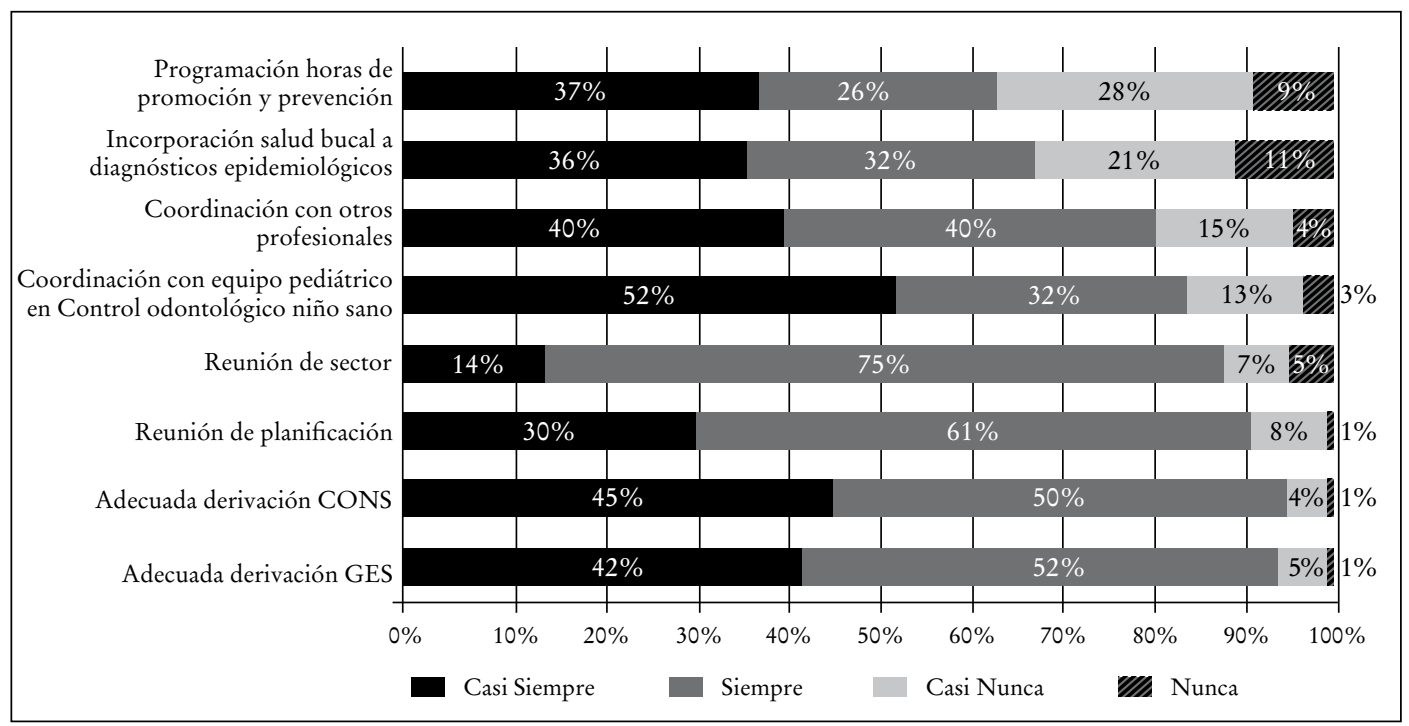

Gráfico $N^{\circ}$ 3: Participación de actividades con la comunidad de los equipos odontológicos encuestados*

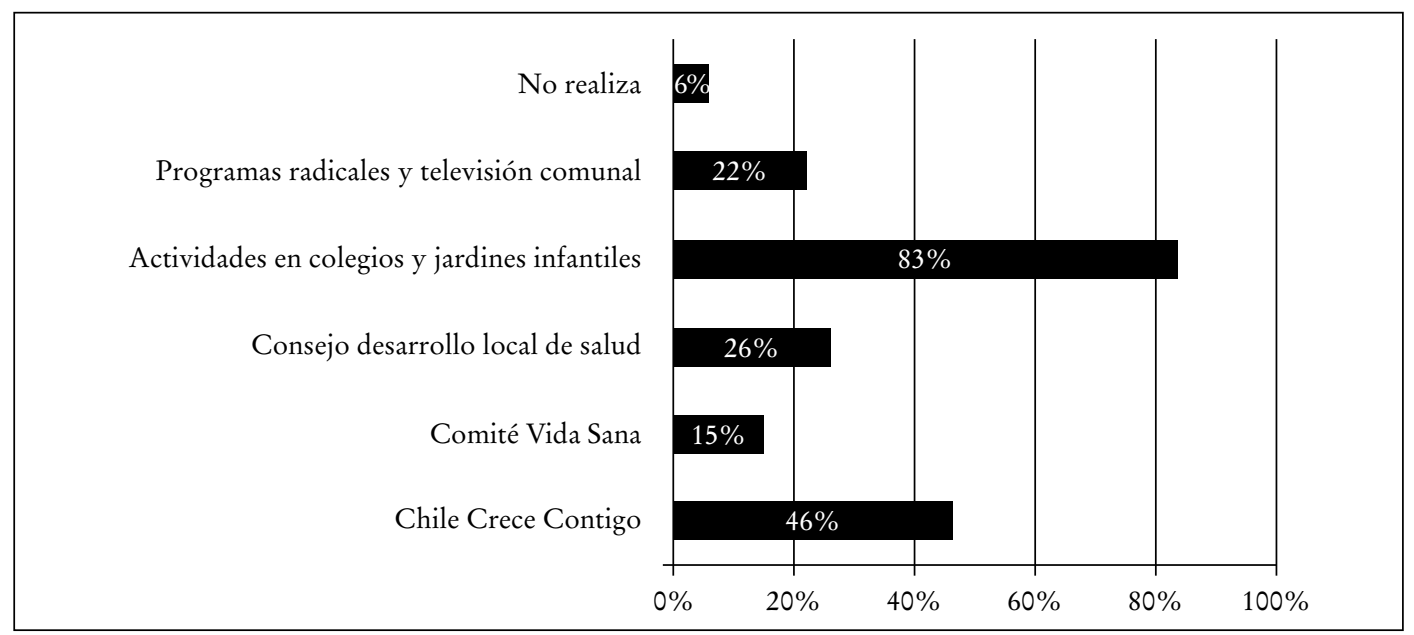

* Respuestas incluyeron más de una alternativa, por lo que el total puede superar el 100\% 
liza en incorporar temas odontológicos; $33 \%$ de los diagnósticos epidemiológicos nunca o casi nunca incorpora algún tema de salud bucal. Esto provoca que problemas de salud bucal no se aborden con un enfoque familiar, al no existir un diagnóstico adecuado que permita caracterizar la población y detectar factores de riesgo asociados, proceso que antecede a diversas actividades de promoción y prevención exitosas (14-16). Además, diversos estudios han documentado que factores sociales como nivel educacional, nivel socioeconómico y ruralidad pueden determinar un mayor riesgo e incidencia de historia de caries, por lo que se deben considerar en la planificación de las estrategias del programa odontológico (17-19).

Otro aspecto analizado fue la participación dentro del equipo de salud y la comunidad; la autopercepción en general fue positiva, pero disminuye frente a la incorporación de otros profesionales a los programas odontológicos, resultados que son compartidos por otros estudios. Evaluaciones al control odontológico del niño sano han demostrado una escasa participación de médicos, enfermeras u otros profesionales en la planificación y entrega de estas prestaciones (20-22). Además se observa una baja participación en programas que tienen como objetivo actuar sobre factores determinantes de la salud bucal, como es el caso del programa Chile Crece Contigo; en el año 2009 la salud bucodental representó solo el 5,7 de los ingresos a educación grupal y dentro de los objetivos del programa de desarrollo integral del niño no se incluyen temas odontológicos $(23,24)$.

Por otra parte, se observa que la mayoría realiza actividades de promoción y prevención $(80 \%)$, principalmente en establecimientos educacionales, donde generalmente se utiliza la educación para la salud como estrategia y tiene como objetivo provocar un cambio de conducta individual, como lo es un correcto cepillado de dientes o una dieta saludable. Sin embargo estas intervenciones se traducen en cambios conductuales a corto plazo, y por lo tanto intervenir en las familias se transformaría en un agente motivador, permitiendo perpetuar esfuerzos de tiempo y recursos en una conducta saludable (14-17,
19). En este contexto, se observa que un porcentaje importante de los equipos odontológicos, realiza consejerías familiares o visitas domiciliarias (14\% y $32 \%$, respectivamente), actividades que debieran seguir estimulándose y no limitar las estrategias de promoción solo a la educación en colegios, sino incluir en éstas a la comunidad y en especial a la familia.

Con respecto a la incorporación al nuevo modelo, la mayoría lo considera positivo; sin embargo se identifican dificultades en su total integración, siendo la falta de recursos humanos y físicos su principal barrera. Además el hecho de que el programa odontológico sea visto como transversal provoca que los odontólogos no sectoricen sus prestaciones. Sin embargo un $65 \%$ de los centros que no realizan atención sectorizada tiene un financiamiento per cápita urbano, con un promedio de 5,4 odontólogos por equipo, lo que puede ser reflejo de un problema de gestión más que de recursos humanos.

Por otra parte, un $70 \%$ considera necesaria una protocolización del programa odontológico con enfoque familiar, de manera de estandarizar y objetivar las prestaciones que deben ser incorporadas, mejorando el sistema de registro, ya que en algunas ocasiones, como el Resumen Estadístico Mensual en su categoría 19, que cuantifica las actividades de promoción, no existe la opción para que los odontólogos puedan hacerlo, generando que estas acciones no se cuantifiquen y por tanto no se valoricen (25).

En este sentido, las estrategias de acción no se deben reducir al equipo odontológico, sino que debe incorporar a los demás profesionales de la salud, aprovechando instancias donde convergen otros actores sociales, como en el caso de los consejos locales de salud o los comités Vida Sana, que llevan años desarrollando estrategias de promoción y prevención para enfrentar problemas de salud que afectan directamente la salud oral (26-28).

\section{Limitaciones del estudio}

Dentro de las limitaciones del estudio, encontramos que la información obtenida vía e-mail, puede tener sesgos y no incluir 
aspectos cualitativos de importancia en caracterizar la implementación del modelo. Además no se incluyó los centros de salud que no estaban acreditados $\mathrm{u}$ hospitales comunitarios de la Atención Primaria, los cuales poseen otra realidad en la implementación del modelo.

\section{AGRADECIMIENTOS}

Al Departamento de Salud Bucal de la Subsecretaría de Salud Pública del MINSAL, por su gestión en la recolección de la información.

\section{BIBLIOGRAFÍA}

1. Chile. Ministerio de Salud. En el camino a Centro de Salud Familiar. Santiago: MINSAL, Subsecretaría de Redes Asistenciales; 2008.

2. Chile. Ministerio de Salud. Marco referencial hospitales de la familia y la comunidad. Santiago: MINSAL, Subsecretaría de Redes Asistenciales; 2009.

3. Hidalgo CG, Carrasco E. Salud familiar: un modelo de atención integral en la atención primaria. $2^{\mathrm{a}}$ edición. Santiago: PUC; 2002.

4. Chile. Ministerio de Salud. Guías clínicas salud oral integral para niños y niñas de 6 años. Santiago: MINSAL, Departamento de Salud Bucal; 2008.

5. Chile. Ministerio de Salud. Guía clínica salud oral integral para embarazadas. Santiago: MINSAL, Departamento de Salud Bucal; 2008.

6. Chile. Ministerio de Salud. Estrategia Nacional de Salud 2011-2020. MINSAL; 2011.

7. León M, Zillmann G. Evaluación bucodental en niños de 0 a 3 años realizada en el Control de Salud del Niño en Consultorios y CESFAM, Región Metropolitana, Santiago-Chile [tesis]. Santiago: Universidad de Chile, Facultad de Odontología; 2009.

8. Chile. Ministerio de Salud. Programa educativo de promoción y prevención en salud bucal para niños y niñas preescolares. San- tiago: MINSAL, Departamento Salud Bucal; 2007.

9. Tristan-Lopez A. Modificación al modelo de Lawshe para el dictamen cuantitativo de la validez de contenido de un instrumento objetivo. Avances en medición. 2008; 6(1): 37-48.

10. Henríquez O, Espinoza M. Capacitación en salud familiar [ppt]. I Congreso Salud Familiar y Atención Primaria; 2008 6-8 nov.; Viña del Mar, Chile.

11. Kravet S, Shore A, Miller R, Green G, Kolodmer K, S Wrigth. Health care utilization and the proportion of primary care physicians. Am J Med. 2008 Feb;121(2):142-8.

12. Mercado R. Caracterización y análisis del perfil de derivaciones de un centro de salud familiar al nivel secundario [tesis]. Santiago: PUC. Departamento Medicina Familiar; 2010.

13. Peñaloza B, Leisewitz T, Bastías G, Zárate V, Depaux R, Villarroel L, et al. Metodología para evaluación de la relación costoefectividad en centros de atención primaria de Chile. Rev Panam Salud Pública. 2010; 28(5): 376-387.

14. Pontificia Universidad Católica de Chile. Modelo de atención en salud familiar. Una propuesta para contribuir al cambio. Santiago: PUC, Programa de Medicina Familiar y Comunitaria; 2001.

15. Shearer DM, Thomson WM, Broadbent JM, Poulton R. Does maternal oral health predict child oral health-related quality of life in adulthood? Health Qual Life Outcomes. 2011; 9: 50.

16. Colussi CF; Calvo MCM. Modelo de avaliação da saúde bucal na atenção básica. Cad Saúde Pública. 2011; 27(9).

17. Ismail AI, Ondersma S, Jedele JM, Little RJ, Lepkowski JM. Evaluation of a brief tailored motivational intervention to prevent early childhood caries. Community Dent Oral Epidemiol. 2011 Oct.; 39(5): 433-48.

18. Tyagi R. The prevalence of nursing caries in Davangere preschool children and its relationship with feeding practices and socioeconomic status of the family. I Indian Soc Pedod Prev Dent. 2008 Dec.; 26(4): 153-7. 
19. Fuenzalida M, Cobs V, Letelier MJ, Del Valle C. Evaluación de enfoques de programas del Departamento de Salud Bucal del Ministerio de Salud mediante sistema de información geográfica. En: Libro de Resumenes XXIX Jornadas Chilenas de Salud Pública. Santiago: Universidad de Chile, Facultad de Medicina, Escuela de Salud Pública; 2010.

20. Abarca I, Arteaga O, et al. Evaluación de calidad del control odontológico del niño sano de 2 y 4 años en centros de atención primaria de la región del Maule, año 2010. Santiago: Trabajo de investigación, Requisito para optar al grado de Magíster en Salud Pública. Facultad de Medicina, Universidad de Chile; 2010.

21. Ceballos M, Acevedo C, Corsini G, Jans A. Diagnóstico en salud bucal de niños de 2 y 4 años que asisten a la educación preescolar en la región Metropolitana. Santiago: MINSAL, Departamento Salud Bucal; 2007.

22. Soto L, Tapia R, Jara G, Rodríguez G. Diagnóstico en salud bucal de los niños de $2 \mathrm{y}$ 4 años de edad que asisten a la educación preescolar en la zona norte y centro del país.
Santiago: MINSAL, Departamento Salud Bucal; 2009.

23. Chile. Ministerio de Planificación. Cuatro años creciendo juntos: memoria de la instalación del Sistema de Protección Integral a la Infancia Chile Crece Contigo 2006-2010. Santiago: MIDEPLAN; 2011.

24. Chile. Programa Protección Social Chile Crece Contigo. Santiago: Programa de apoyo Biopsicosocial. Catálogo de prestaciones; 2012

25. Chile. Ministerio de Salud. Resumen Estadístico Mensual. Serie A versión 1.5. Santiago: MINSAL, Departamento de Estadística e Informaciones en Salud; 2011.

26. Chile. Ministerio de Salud. Propuesta de trabajo, Estrategia Global contra la obesidad. Santiago: MINSAL; 2006.

27. Salinas J, Cancino A, Pezoa S, Salamanca F, Soto M. Vida Chile 1998-2006: resultados y desafíos de la política de promoción de la salud en Chile. Rev Panam Salud Pública. 2007; 21(2/3): 136-144.

28. Peroni A. El desarrollo local a escala humana: experiencias de desarrollo comunitario en el sector salud. Chile. Rev Univ Bolivariana. 2009; 8(22): 99-120. 